\title{
SUSTAINABLE PROCESS FOR SPARTEINE SULFATE PREPARATION
}

Ignacio Regla*a, Manuel López-Ortiza , Otoniel Jiméneza, Simón Hernández-

Ortega $^{\mathrm{b}}$, Astrid Ramírez-Betancourt ${ }^{\mathrm{c}}$ and Kalina Bermúdez-Torres ${ }^{\star c}$

aFacultad de Estudios Superiores-Zaragoza, UNAM, Batalla del 5 de Mayo esq. Fuerte de Loreto, Ejercito de Oriente, 09230 México, D.F., México E-mail: regla@unam.mx

' Instituto de Química, UNAM, Av. Universidad 3000, 04510 Ciudad Universitaria. Coyoacán CDMX. 'Instituto Politécnico Nacional-Centro de Desarrollo de Productos Bióticos, Departamento de Biotecnología. Calle CeProBi No. 8, Col. San Isidro, Yautepec, Morelos, México. C.P. 62731. kbermudes@ipn.mx

\section{Supporting Information}

\section{Contents}

General information .............................................................................................

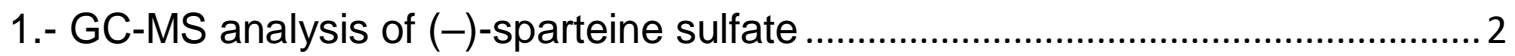

Figure $S 1$. Gas chromatogram of (-)-sparteine sulfate prepared from $L$. montanus.

Figure S2. Mass spectrum of (-)-sparteine sulfate prepared from L. montanus. 4

Figure S3. Gas chromatogram of (-)-sparteine from Aldrich $\AA$............................. 5

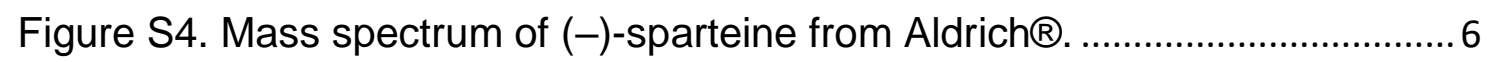

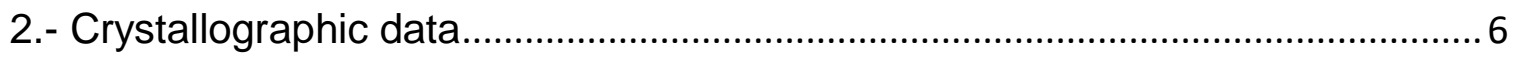

Table S1. Crystal data and structure refinement for (-)-sparteine sulfate ........... 6

\section{General information}

Leaves and stems of L. montanus were collected at the Iztaccíhuatl-Popocatépetl National Park and stored immediately after arrival in the laboratory at $-22{ }^{\circ} \mathrm{C}$. Having in mind sustainability precepts, only about ten percent of the total population of the plants in the park were collected.

Ammonium hydroxide and sulfuric acid were technical grade, purchased from Reactivos Meyer, Cyclopentyl methyl eter, ReagentPlus ${ }^{\circledR}$ was purchased from Aldrich. 


\section{1.- GC-MS analysis of (-)-sparteine sulfate}

The gas chromatography was performed in a gas chromatograph (Agilent 7890A $\mathrm{CG}$ ) coupled to a mass spectrometer (Agilent 5975C MSD) with electronic impact detector $(70 \mathrm{eV})$.

$1 \mu \mathrm{L}$ of a methanolic solution of obtained sparteine sulfate $(1 \mathrm{mg} / \mathrm{mL}+20$ microliters $/ \mathrm{mL}$ concentrated ammonium hydroxide) was automatically injected in the gas chromatograph. A non-polar fused silica capillary column, with a length of $30 \mathrm{~m}$, inner diameter $0.25 \mathrm{~mm}$ and film thickness $0.25 \mu \mathrm{m}$ (SUPELCO Analytical) was used to separate the compound; hydrogen was used as the carrier gas at a flow rate of $1.5 \mathrm{~mL} / \mathrm{min}$ (SPLIT mode) and an injector temperature of $280{ }^{\circ} \mathrm{C}$. The initial temperature was $120^{\circ} \mathrm{C}$ for two minutes in isothermal flow, followed by an increase in the linear isothermal temperature to $300{ }^{\circ} \mathrm{C}$, at a speed of $8^{\circ} \mathrm{C} \mathrm{min}-1$, for 10 minutes in isothermal mode at $300^{\circ} \mathrm{C}$. The peaks of the gas chromatogram were integrated and their corresponding mass spectra were compared to the NIST spectrum library (NIST/EPA/NIH, 2002). 
Abundance

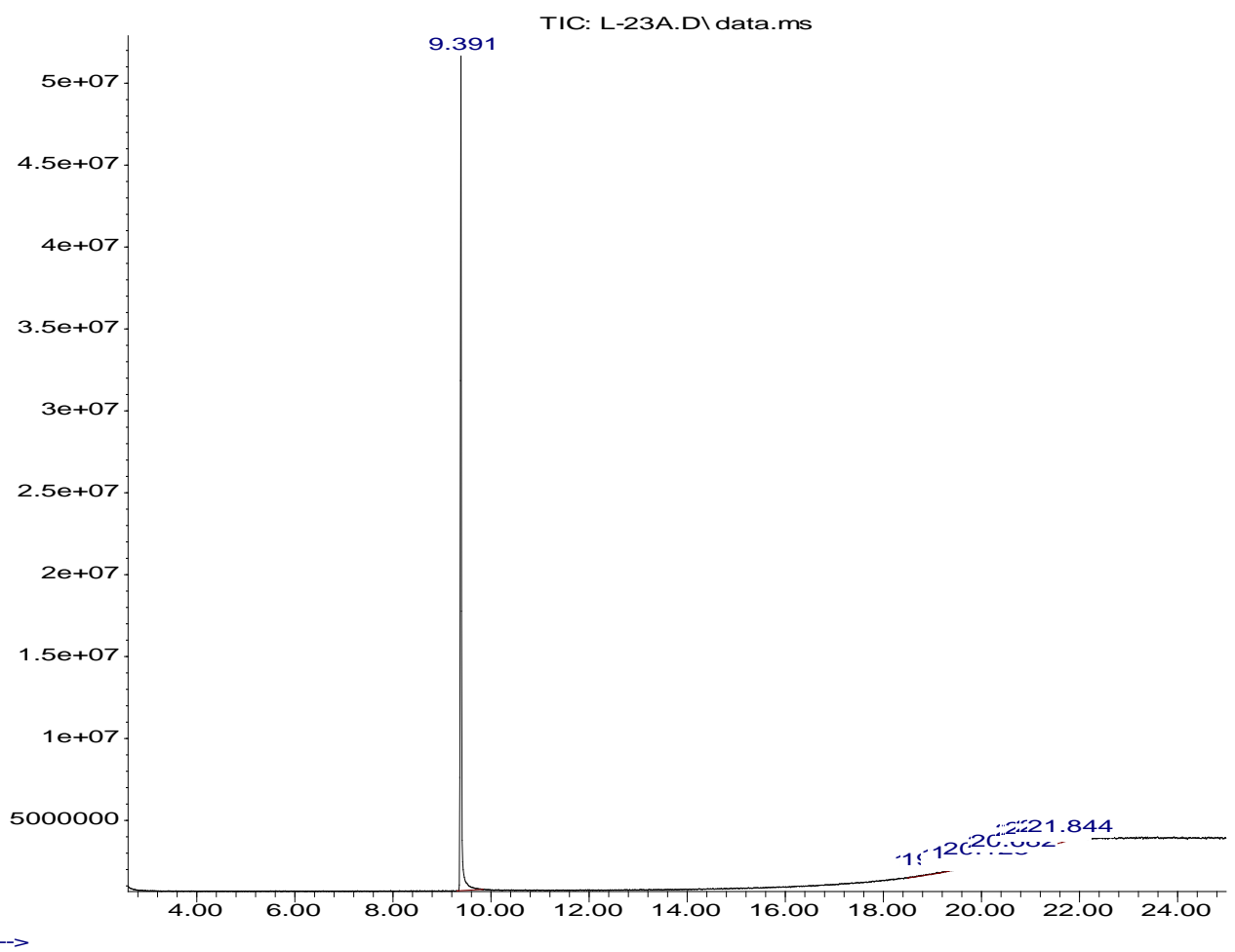

Time-->

\begin{tabular}{|c|c|c|c|c|c|}
\hline Peak \# & Ret Time & Type & Width Area & Start Time & End Time \\
\hline 1 & 9.391 BV & 0.059 & 830843719 & 9.308 & \\
\hline 2 & 19.109 PV & 0.137 & 6240596 & 18.50019 .130 & \\
\hline 3 & 19.256 PV & 0.049 & 1399462 & 19.13019 .303 & \\
\hline 4 & 19.670 PV & 0.161 & 1652947 & 19.30319 .707 & \\
\hline 5 & $19.818 \mathrm{VV}$ & 0.039 & 1357482 & 19.70719 .843 & \\
\hline 6 & 19.873 PV & 0.017 & 317336 & 19.84319 .888 & \\
\hline 7 & 20.128 PV & 0.150 & 4965925 & 19.88820 .146 & \\
\hline 8 & 20.622 PV & 0.188 & 18540416 & 20.14620 .638 & \\
\hline 9 & 20.682 VV & 0.050 & 3937686 & 20.63820 .698 & \\
\hline 10 & $21.190 \mathrm{VV}$ & 0.259 & 71299117 & 20.69821 .234 & \\
\hline 11 & $21.296 \mathrm{VV}$ & 0.076 & 13887421 & 21.23421 .316 & \\
\hline 12 & $21.338 \mathrm{VV}$ & 0.034 & 6491497 & 21.31621 .353 & \\
\hline 13 & $21.387 \mathrm{VV}$ & 0.051 & 10149091 & 21.35321 .413 & \\
\hline 14 & $21.600 \mathrm{VV}$ & 0.165 & 32297131 & 21.41321 .640 & \\
\hline 15 & $21.684 \mathrm{VV}$ & 0.068 & 9855796 & 21.64021 .737 & \\
\hline 16 & 21.844 VV & 0.103 & 5678461 & 21.73721 .869 & Library Search Report \\
\hline
\end{tabular}

Figure S1. Gas chromatogram of (-)-sparteine sulfate prepared from L. montanus 
Abundance

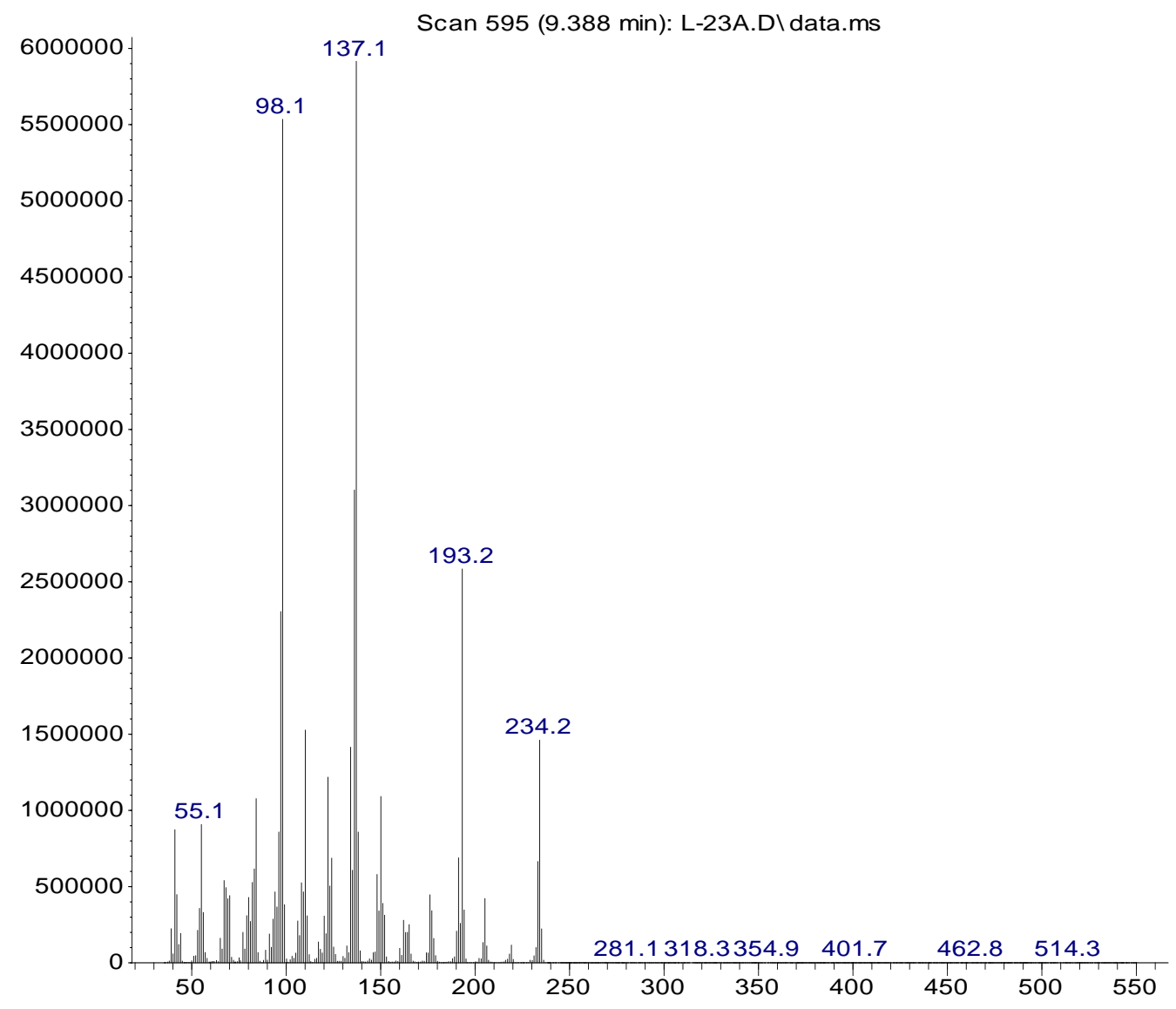

$\mathrm{m} / \mathrm{z-->}$

Figure S2. Mass spectrum of (-)-sparteine sulfate prepared from L. montanus. 
Abundance

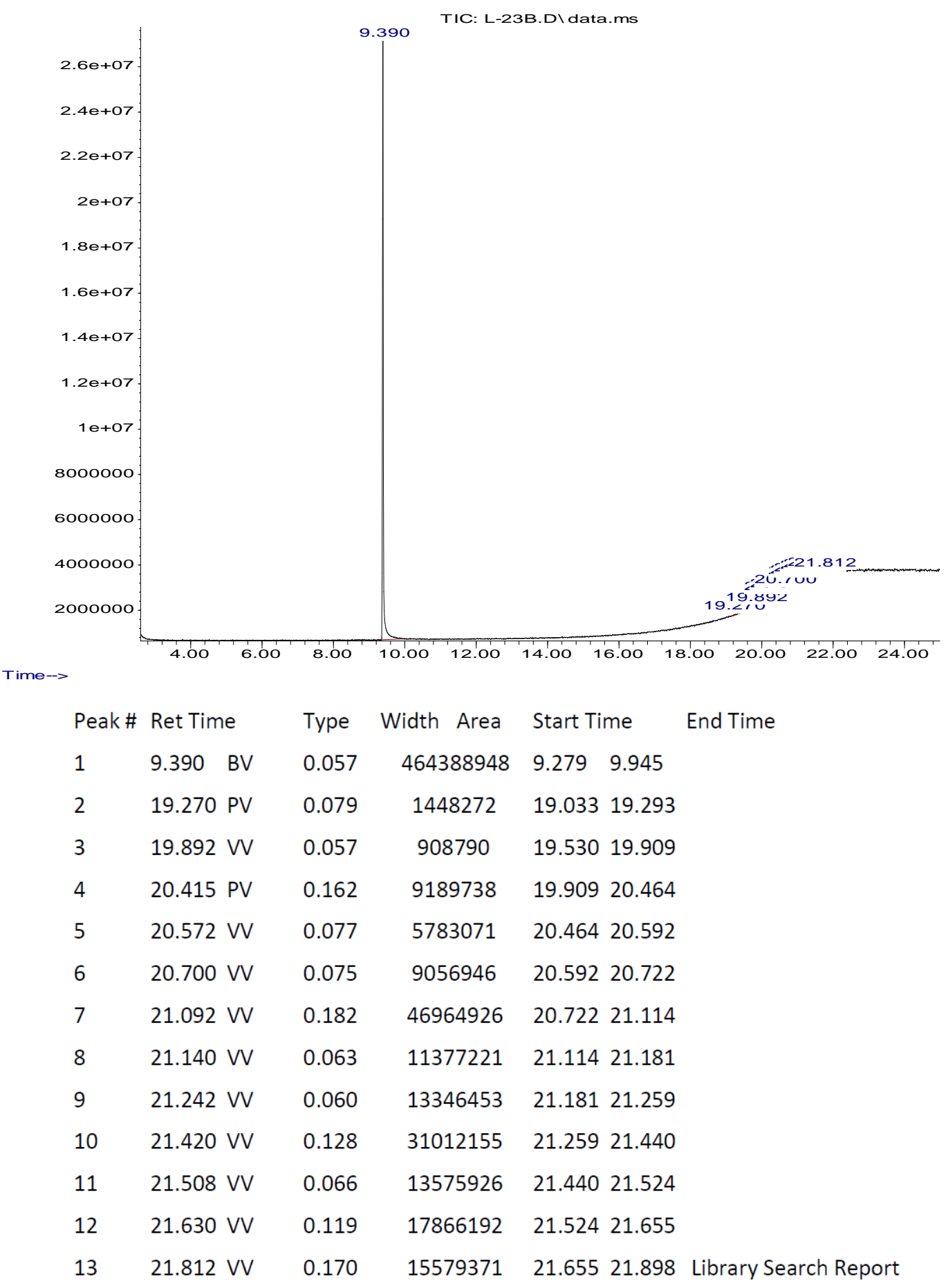

Figure S3. Gas chromatogram of (-)-sparteine from Aldrich $\AA^{\circledR}$. 


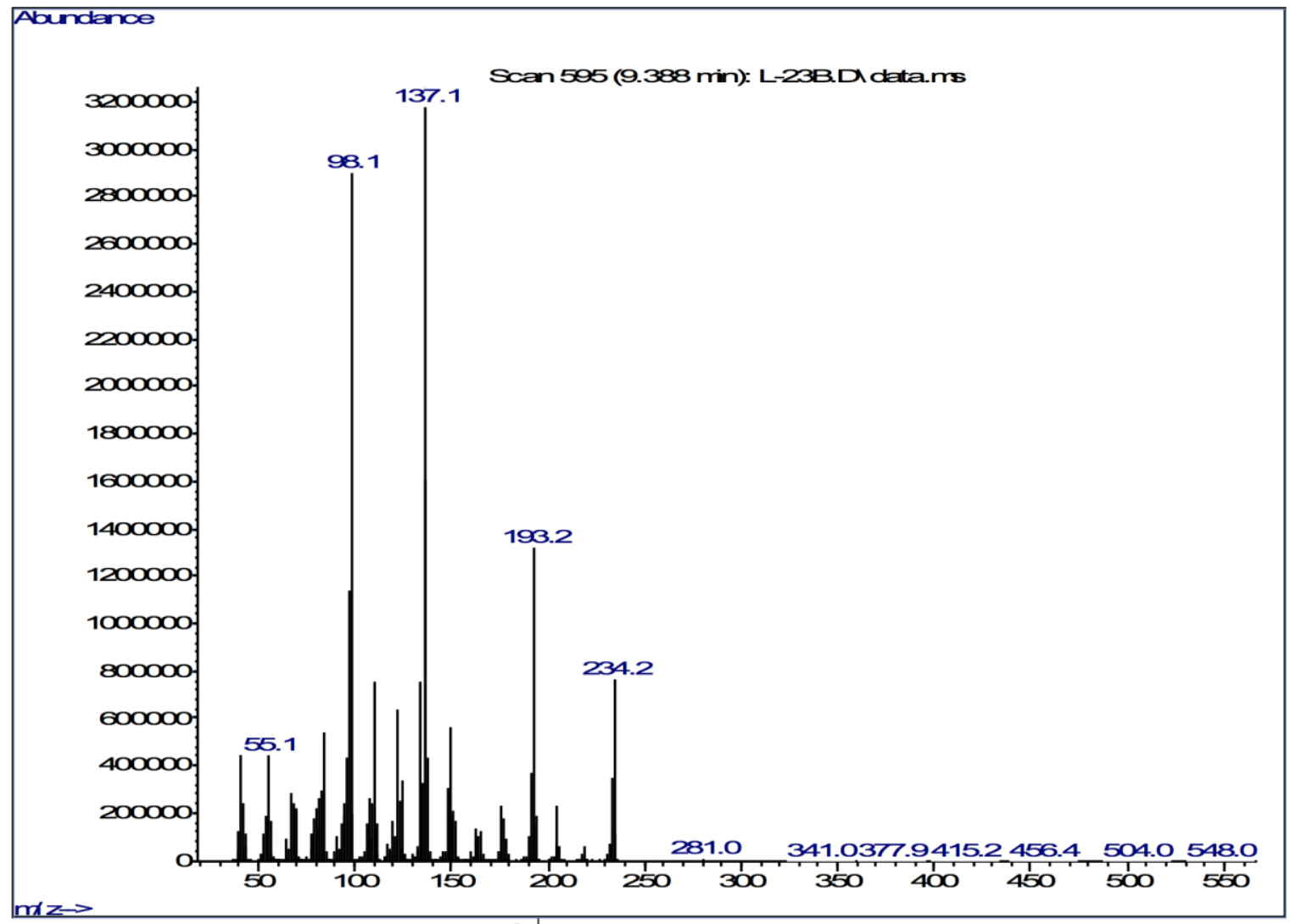

Figure S4. Mass spectrum of (-)-sparteine from Aldrich®.

\section{2.- Crystallographic data}

Crystallographic data was obtained in a Bruker Smart Apex II diffractometer with a Cu-target $X$-ray source $(\lambda=1.54178 \AA)$. These data can be obtained free of charge from The Cambridge Crystallographic Data Centre with the deposition number CCDC 1949136.

Table S1. Crystal data and structure refinement for (-)-sparteine sulfate

\begin{tabular}{|l|l|}
\hline Identification code & $330 \mathrm{CPI} 19$ \\
\hline Empirical formula & $\mathrm{C} 15 \mathrm{H} 38 \mathrm{N2}$ O9 S \\
\hline Formula weight & 422.53 \\
\hline Temperature & $150(2) \mathrm{K}$ \\
\hline Wavelength & $1.54178 \AA$ \\
\hline Crystal system & Monoclinic \\
\hline Space group & P21 \\
\hline
\end{tabular}




\begin{tabular}{|c|c|c|}
\hline \multirow[t]{3}{*}{ Unit cell dimensions } & $a=7.9580(2) \AA$ & $\alpha=90^{\circ}$ \\
\hline & $\mathrm{b}=15.1831(3) \AA$ & $\beta=92.0530(10)^{\circ}$. \\
\hline & $c=8.8246(2) \AA$ & $y=90^{\circ}$. \\
\hline Volume & 1065.57(4) Å3 & \\
\hline $\mathbf{Z}$ & \multicolumn{2}{|l|}{2} \\
\hline Density (calculated) & \multicolumn{2}{|l|}{$1.317 \mathrm{Mg} / \mathrm{m} 3$} \\
\hline Absorption coefficient & \multicolumn{2}{|l|}{$1.769 \mathrm{~mm}-1$} \\
\hline$F(000)$ & \multicolumn{2}{|l|}{460} \\
\hline Crystal size & \multicolumn{2}{|c|}{$0.491 \times 0.369 \times 0.102 \mathrm{~mm} 3$} \\
\hline $\begin{array}{l}\text { Theta range for data } \\
\text { collection }\end{array}$ & \multicolumn{2}{|c|}{5.015 to $68.274^{\circ}$} \\
\hline Index ranges & \multicolumn{2}{|c|}{$\begin{array}{l}\text { Index ranges }-9<=h<=9,-18<=k<=18,- \\
10<=\mid<=10\end{array}$} \\
\hline Reflections collected & \multicolumn{2}{|c|}{13465} \\
\hline Independent reflections & \multicolumn{2}{|c|}{$3894[R$ (int) $=0.0289]$} \\
\hline $\begin{array}{l}\text { Completeness to theta }= \\
67.679^{\circ}\end{array}$ & \multicolumn{2}{|c|}{$100.00 \%$} \\
\hline Absorption correction & \multicolumn{2}{|c|}{ Semi-empirical from equivalents } \\
\hline Max. and min. transmission & \multicolumn{2}{|c|}{0.7531 and 0.5803} \\
\hline Refinement method & \multicolumn{2}{|c|}{ Full-matrix least-squares on F2 } \\
\hline Data / restraints / parameters & \multicolumn{2}{|c|}{$3894 / 11 / 280$} \\
\hline Goodness-of-fit on F2 & \multicolumn{2}{|l|}{1.061} \\
\hline Final $R$ indices [l>2sigma(I)] & \multicolumn{2}{|c|}{$\mathrm{R} 1=0.0267, \mathrm{wR2}=0.0686$} \\
\hline $\mathbf{R}$ indices (all data) & \multicolumn{2}{|c|}{$R 1=0.0270, w R 2=0.0690$} \\
\hline $\begin{array}{l}\text { Absolute structure } \\
\text { parameter }\end{array}$ & \multicolumn{2}{|c|}{$0.070(6)$} \\
\hline Largest diff. peak and hole & \multicolumn{2}{|c|}{0.363 and -0.231 e. $\AA \dot{A}-3$} \\
\hline
\end{tabular}

